\title{
Cathode spot initiation under plasma
}

\author{
V F Puchkarev and M B Bochkarev \\ Russian Academy of Sciences, Institute of Electrophysics, Yekaterinburg 620219, \\ Komsomolskaya 34, Russia
}

Received 17 May 1993, in final form 25 February 1994

\begin{abstract}
By using the procedure of current detection from individual cathodes 5-7 $\mu \mathrm{m}$ distant from each other and by scanning electron microscopy, the initiation of new vacuum arc cathode spots has been studied. Ion current bursts of duration 10-50 ns with an amplitude of 0.1-1 A, and inceptive craters on the initially virgin cathode, have been detected just before the spot transition from one cathode to another. The current density estimated over inceptive craters is $>10^{11} \mathrm{~A} \mathrm{~m}^{-2}$. The power flux density of the jet from plasma onto the cathode exceeds $10^{12} \mathrm{~W} \mathrm{~m}^{-2}$ and is sufficient to heat the cathode surface up to the boiling point for a time $\simeq 1$ ns. The mechanism of spot initiation and its motion in a transverse magnetic field is discussed.
\end{abstract}

\section{Introduction}

The motion of a cathode spot over the cathode surface rather than its operation on the originally heated place is its most mysterious feature. As a matter of fact, this motion is the appearance of a new spot and the death of a previous one [1-3]. Therefore, the mechanism of spot motion reduces to the problem of new spot initiation. From this point of view, retrograde motion is the result of preferable initiation of a new cathode spot in the direction of maximum magnetic field [3]. In the magnetic field the stability of an arc increases [1], and this, in it's turn, is a consequence of the fact that a spot lives longer on the 'retrograde side' rather than on the 'direct side' [3].

A lot of publications are dedicated to mechanisms of new cathode spot initiation. Most of them discuss more or less probable models and almost no direct experimental data. This paper presents experimental results on the study of spot initiation with high spatial (a few micrometres) and temporal (a few nanoseconds) resolutions. We also survey existing models of new spot initiation under plasma.

\section{A brief review of mechanisms of spot initiation}

Characteristics of arc operation on a contaminated cathode surface are distinguished very strongly from those on a clean surface. One can find a brief discussion of these features and a spot model in [4]. According to study [5] and study of a glow-to-arc transition mechanism [6], a new spot is initiated under plasma due to breakdown of non-metallic films. Therefore, a trace left by the arc is craters spread $5-50 \mu \mathrm{m}$ distant from each other [7].
On a clean cathode a spot moves step by step with a step approximately equal to the crater radius (figure $1(a))$. The following concepts are the most disputable now.

(i) A new spot is assumed to be initiated on the rim of the crater due to an explosion-like destruction of microprotrusions in the field of plasma space charge. Micropoints appear due to displacement of a liquid layer onto the crater rim by the plasma pressure [8-11]. This mechanism, however, meets some difficulties. The electric field created by plasma space charge is too small (being about $10^{9} \mathrm{~V} \mathrm{~m}^{-1}$ ) to explode micropoints during nanosecond time periods. The assumption that the field is enhanced at the moments of arc voltage bursts (if the amplitude is higher than $100 \mathrm{~V}$ ) has been proved to be invalid: direct experiment has shown that step by step motion is accompanied only by low-voltage $(5-10 \mathrm{~V})$ fluctuations [12].

(ii) The modified hypothesis [13] relates the condition of new spot initiation to the moment when a droplet detaches from a liquid protrusion. The current density is increased many fold in the contraction region providing fast explosion in this narrow spot at 'the neck'. For example, to explode 'the neck' during $1 \mathrm{~ns}$ (which seems to be the maximum value, because of high velocity of molten layer motion $v_{1} \simeq 100 \mathrm{~m} \mathrm{~s}^{-1}$ ) the current density flowing through 'the neck' must be as high as $10^{13} \mathrm{~A} \mathrm{~m}^{-2}$. As the current density of ions and returning electrons from plasma does not exceed $10^{9} \mathrm{~A} \mathrm{~m}^{-2}$, the enhancement factor should be about 100 . This is questionable, since the most probable droplet diameter is $2 r_{\mathrm{d}}=0.1 \mu \mathrm{m}$. Hence, the radius of 'the neck' should be $r_{\mathrm{n}}=0.02 \mu \mathrm{m}$, and a characteristic time of existence of 'the neck' is $\tau \leqslant r_{n} / \nu_{1} \simeq 0.1 \mathrm{~ns}$.

(iii) The two models above are based on explosionlike heating of micropoints. Another model proceeds 
(a)

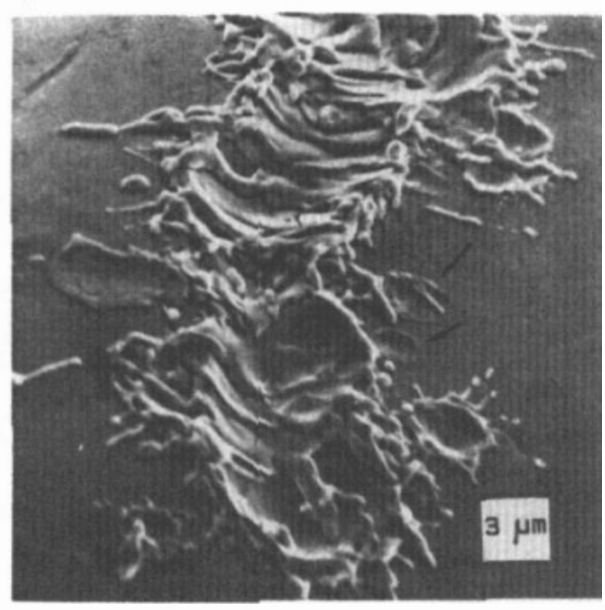

(b)

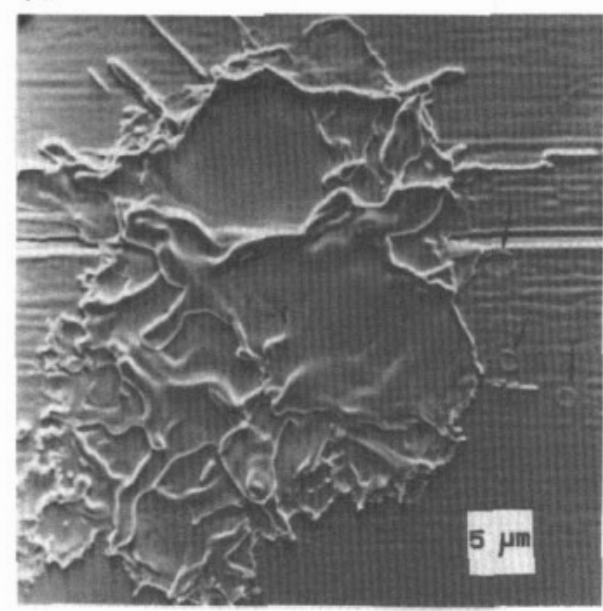

Figure 1. Traces left by arc on a tungsten cathode. Arrows shows incipient craters: (a) arc current $10 \mathrm{~A}$; (b) arc current $20 \mathrm{~A}$.

from the fact that Joule heating and thermal runaway result in an increase of the voltage drop in the cathode body (and the near-cathode plasma) until a new spot appears on the crater rim [14]. This allowed the formula for the spot velocity to be derived as $V_{\mathrm{s}}=$ $2 K\left(J / I_{\mathrm{a}}\right)^{1 / 2}$, where $K, J$ and $I_{\mathrm{a}}$ are thermal diffusivity, current density and arc current, respectively. Experiment shows, however, that the spot velocity is proportional to the root of the arc current [12]. Nemchinsky [15] connects the spot velocity with the amplitude of the arc voltage fluctuation $V_{\mathrm{s}} U_{\mathrm{a}}^{-1}$. Since the amplitude $U_{\mathrm{a}}$ at small current decreases as the current is increased, this formula explains the current's dependence on spot velocity. However, as we have discovered in [12], spot motion is accompanied by small fluctuations in the are voltage $\left(U_{\mathrm{a}} \simeq 5-10 \mathrm{~V}\right)$ also at the minimum current, that is, it is independent of voltage fluctuations.

(iv) Another model proceeds from the following concept [16]. Plasma potential increases continuously in the region of metal-to-plasma transition. High electron temperature $(5 \mathrm{eV})$ gives rise to circulation of current at the expense of returning electrons from the plasma to the cathode. The amplitude of this current can surpass the arc current flowing to the anode (the unipolar

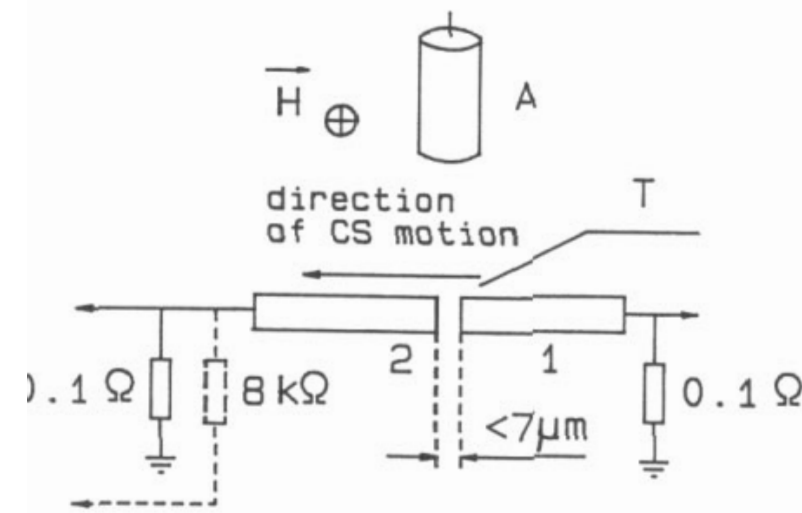

Figure 2. Experimental set-up. A, anode; $T$, trigger pin; and 1 and 2 , cathodes. ( $8 \mathrm{k} \Omega$ was used for the floating regime.)

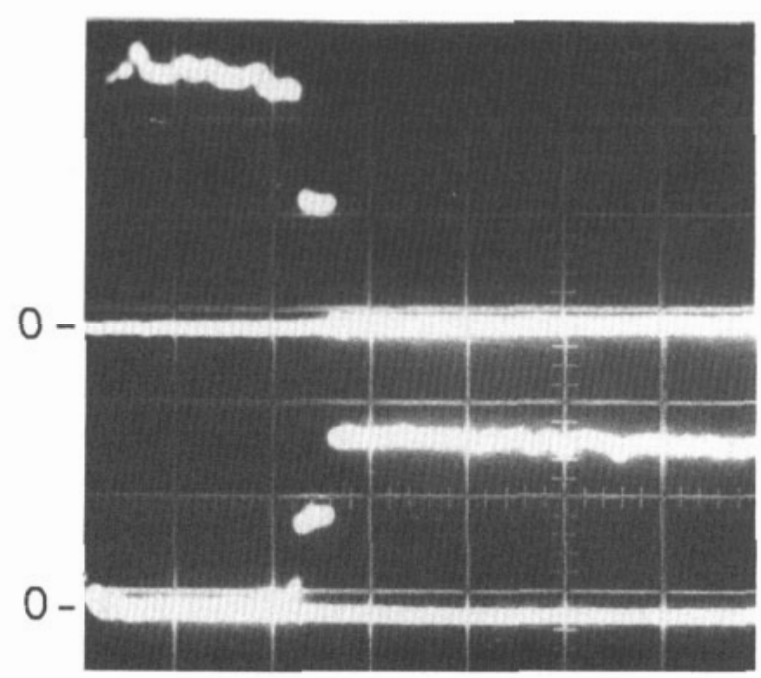

Figure 3. Waveforms of arc current at the moment of transition: upper trace, cathode 1 ( $3 \mathrm{~A}$ per division); lower trace, cathode 2 (5 A per division); time 200 ns per division.

effect). A high density of power flux transferred by these electrons can ignite a new spot for a few nanoseconds [12]. Inceptive craters a few micrometres distant from the main spot track (figure 1) can be formed by the returning electron flux. The existence of such craters has stimulated experimental verification of this idea. In addition, Juttner et al [17] have shown by experiment with a spot moving across the slit, that the cathode spot can jump over the slit even on a clean surface, if the space between the slit edges is less than $10 \mu \mathrm{m}$. Hence, there exists a mechanism of new spot ignition at distance a little larger than the crater radius.

\section{Experiment}

As cathode we used a thin tungsten wire $50-100 \mu \mathrm{m}$ in diameter divided into two parts (figure 2). The cathode surface was smoothed by electropolishing. Before the experiment the electrodes were cleaned by heating at $2000 \mathrm{~K}$ in an oil-free ultra-high vacuum under 
pressure lower than $10^{-6} \mathrm{~Pa}$. The accuracy of the gap setting between the cathodes was limited by microscope resolution, but the gap was always less than 5-7 $\mu \mathrm{m}$.

A cathode spot was initiated by a 50 ns trigger pulse (current about $5 \mathrm{~A}$ ) at a distance of $0.05-0.1 \mathrm{~mm}$ from the end of cathode 1 and was directed by the magnetic field $H \simeq 0.1-0.3 \mathrm{~T}$ to the second cathode. The pulse duration was $3 \mu \mathrm{s}$ and was determined by the length of the RF cable. This time was sufficient for the spot to reach the end of the cathode, as the spot velocity under these conditions was $30-100 \mathrm{~m} \mathrm{~s}^{-1}$ [12]. A twobeam storage oscilloscope with rise time $5 \mathrm{~ns}$ was used to detect current from the electrodes. The arc current varied in the range $5-20 \mathrm{~A}$. The anode $(0.4 \mathrm{~mm}$ in diameter) was spaced $0.5 \mathrm{~mm}$ from the cathodes.

We also studied the influence of the probe location with respect to the cathode spot on the indication of floating potential (see figure 6 later). A probe $0.07 \mathrm{~mm}$ in diameter with non-insulated part $0.1 \mathrm{~mm}$ was placed $0.1 \mathrm{~mm}$ distant from the cathode. Floating potential has been measured for two probe locations: (i) when it was in the cathode shade and (ii) when the ion flux affects the probe. The arc current was $5 \mathrm{~A}$.

\section{Results}

When the cathode surface was not clean enough, the cathode spot appeared on the second cathode in $200-500 \mathrm{~ns}$, namely at the moment when plasma arrived at cathode 2 . On heating the electrodes, the probability of arc transition to the second cathode decreased to $0.1-0.01$, which was defined by the arc current and by the gap between the cathodes. Figure 3 shows oscillograms at the moment of arc transition from one cathode to another. This is a remarkable waveform. First, it shows evidence that there is a probability of initiating a new spot at a distance of a few micrometres from the footpoint of a previous spot on a smooth, clean surface; second, it verifies our former findings, taken by the autograph method [12], that the minimum spot current for a tungsten cathode is about $2 \mathrm{~A}$ and the cathode spot lifetime is $30-50$ ns.

Just prior to spot initiation on the second cathode, we revealed $0.1-1$ A current bursts of positive polarity of duration 10-50 ns. For events when only current bursts were detected (without arc transition) we thoroughly examined the surface of cathode 2 by scanning electron microscopy. Figure 4 shows a micrograph of the cathode 2 surface and waveforms of the arc current and pre-arcing current. The number of current bursts coincides with the number of incipient craters. From the photograph taken at a small angle to the cathode surface one can estimate the depth of these craters as $0.3 \mu \mathrm{m}$. Having transformed the second electrode into a floating probe (figure 2), we measured fluctuations of the floating potential $V_{\mathrm{f}}$ (figure 5 ) and estimated ion energy in the burst $E_{\mathrm{i}}=z V_{\mathrm{f}}$. At a mean ion charge $z=2.7$, for a tungsten cathode $E_{\mathrm{i}}=z V_{\mathrm{f}}=70-160 \mathrm{eV}$. (a)

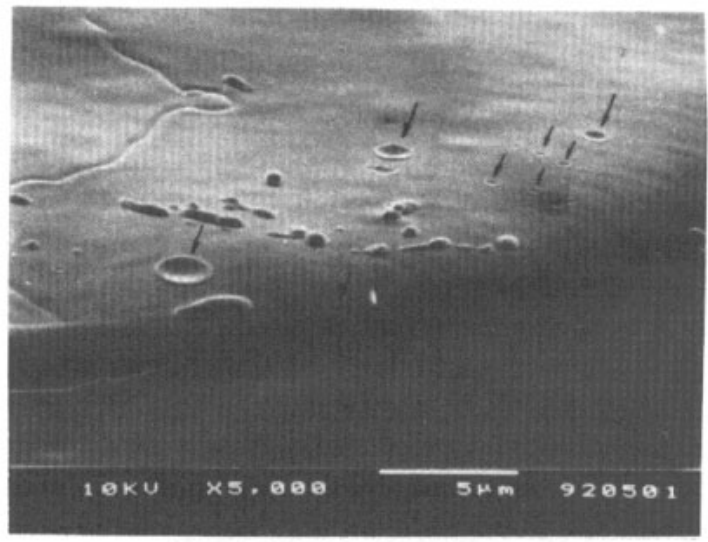

(b)

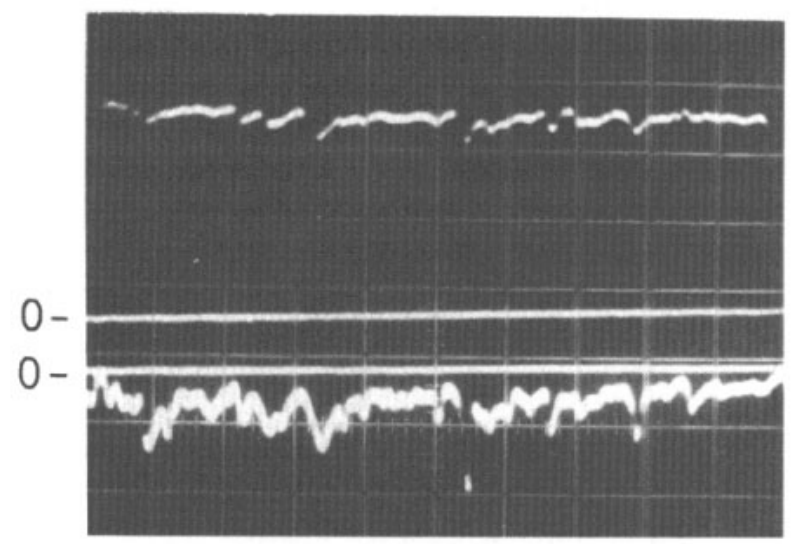

Figure 4. Cathode micrograph (a) and corresponding wavetorms $(b)$ : upper trace, arc current from cathode 1 (1.5 A per division); lower trace, pre-arcing current from cathode 2 (0.5 A per division, inverted signal); time $100 \mathrm{~ns}$ per division.

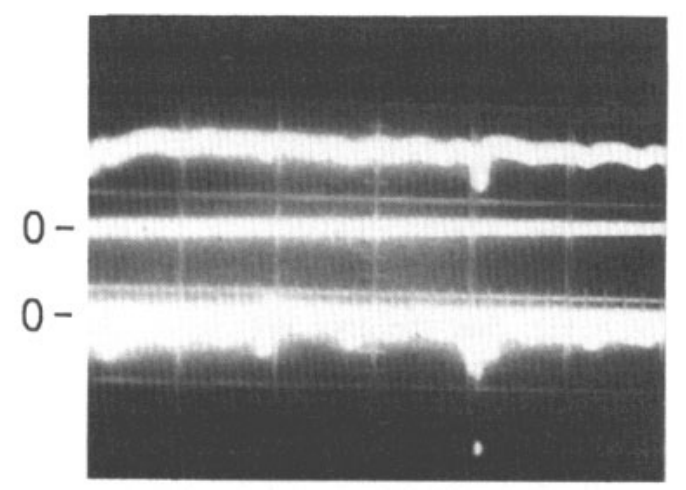

Figure 5. Waveforms of arc current from cathode 1 (upper trace, $5 \mathrm{~A}$ per division) and floating potential from floating probe (lower trace, inverted signal $40 \mathrm{~V}$ per division); time 200 ns per division.

\section{Discussion}

\subsection{The mechanism of spot initiation}

As affected by spot plasma, current bursts preceding spot initiation are detected on the second cathode. On comparing waveforms of the floating potential and the pre-arcing current from cathode 2 , we can assume it to 
be an ion current. The character of crater erosion also shows strong evidence for a surface heating source. We estimated the mean current density over the inceptive crater, assuming that the crater size is proportional to the amplitude of ion current in the burst, as $J \simeq$ $(1-8) \times 10^{11} \mathrm{~A} \mathrm{~m}^{-2}$. The ion current density from the plasma is

$$
J=0.43 z e n_{\mathrm{i}}\left(2 z U_{\mathrm{c}} / m_{\mathrm{i}}\right)^{1 / 2} .
$$

We shall use a formula from [10] to estimate the value of the plasma density $n_{\mathrm{i}}$ at distance $r$ from the centre of the spot:

$$
n_{\mathrm{i}}=\frac{\gamma I_{\mathrm{a}}}{2 \pi m_{\mathrm{j}} v r^{2}}
$$

Here $\gamma$ is the erosion rate and $v$ is the plasma expansion velocity. To obtain current density $J>10^{11} \mathrm{~A} \mathrm{~m}^{-2}$ at $I_{\mathrm{a}}=5 \mathrm{~A}$, the plasma concentration must be as high as $n_{\mathrm{i}}>10^{26} \mathrm{~m}^{-3}$ and $E_{\mathrm{i}}=z U_{\mathrm{c}}=160 \mathrm{eV}\left(U_{\mathrm{c}}\right.$ is the cathode voltage drop; $U_{\mathrm{c}} \simeq V_{\mathrm{f}}=60 \mathrm{~V}$ ). High-energy ions are detected in vacuum arcs [18]. The plasma concentration $n_{i}>10^{26} \mathrm{~m}^{-3}$ at a distance of $5 \mu \mathrm{m}$ from the centre of the plasma source can be calculated for the values $\gamma \geqslant 10^{-3} \mathrm{~g} \mathrm{C}^{-1}$ and $v \simeq 10^{3} \mathrm{~m} \mathrm{~s}^{-1}$. The following facts should also be taken into account when estimating plasma density. Within the spot radius compared with the crater radius, plasma density appears to drop with $r$ slower than an $r^{-2}$ dependence. In the transverse magnetic field the plasma expands at a velocity slower by a factor 3-5 than without the field [19]. Therefore the plasma density from the 'retrograde side' of the spot is higher than that from the 'direct side'. As to the high value of the erosion rate $\gamma \simeq 10^{-3} \mathrm{~g} \mathrm{C}^{-1}$, we measured it in [12] and verified it by collecting and analysing new data [20].

It should be noted that, at such a high plasma density, the electric field in the cathode-plasma layer $E=(k T n / e)^{1 / 2}=3 \times 10^{9} \mathrm{~V} \mathrm{~m}^{-1}$, and the corresponding field emission current $J_{\mathrm{fe}}=10^{5} \mathrm{~A} \mathrm{~m}^{-2}$. Thermionic emission (including the Schottky effect) gives $J_{\mathrm{Te}} \simeq$ $(0.1-5) \times 10^{11} \mathrm{~A} \mathrm{~m}^{-2}$ at cathode temperature $T=$ $(5-6) \times 10^{3} \mathrm{~K}$. We estimate the time $t_{\mathrm{h}}$ required to heat a tungsten cathode up to the boiling point $T_{\mathrm{b}}$ from [21] as

$$
2 Q t^{1 / 2}=T_{\mathrm{b}}(\pi \lambda \rho c)^{1 / 2} .
$$

Here $\lambda, \rho$ and $c$ are the heat conductivity, the specific mass and the heat capacitance respectively. At the flux power density $Q>J U_{\mathrm{c}}=5 \times 10^{12} \mathrm{~W} \mathrm{~m}^{-2}$ the time $t_{\mathrm{h}}<1 \mathrm{~ns}$. The energy deposited into the cathode amounts to about $3 \times 10^{-7} \mathrm{~J}$. This energy provides evaporation of $\simeq 10^{-11} \mathrm{~g}$ of cathode matter. This value corresponds to the volume of the inceptive crater (figure 4 ) and to the value of the erosion rate $\gamma=10^{-3} \mathrm{~g} \mathrm{C}^{-1} m \simeq \gamma q$.

It is remarkable that the inceptive craters are examined both on the top and on the lateral surface of cathode 2 , i.e. independently of cathode 2 orientation with respect to plasma jet direction. This means that, within a radius of about $10 \mu \mathrm{m}$, plasma microjets can be directed both back to the cathode (see figure 1) and to the anode. In addition, the ion current from the plasma is in the form of microjets $\simeq 1 \mu \mathrm{m}$ in diameter. These microjets appear to form due to an increase in the local plasma density, and the ion energy has to surpass the mean value of $z U_{\mathrm{c}}$. Discussing the mechanism of forming high-energy ion jets [22-24], we should bear in mind now that they are microjets rather than a uniform flux. Similar microjets, though in the form of electron microbeams towards an anode with duration 10-20 ns, were detected in the initial stage of the vacuum breakdown [10] when the plasma radius was about $0.2 \mathrm{~mm}$. This evidence also gives the proposal of support microplasmoides, which have a higher density and temperature than the ambient plasma. Such 'hot spots' become detectable in high-current arcs [25]. Fluctuations in local plasma parameters are related to processes at the cathode. These processes cause fluctuations in arc voltage, floating potential and plasma temperature, and they are also responsible for formation of high-energy ion jet bursts [4]. The time scale of these fluctuations is within the nanosecond range.

Now we discuss the role of the returning electron current. During fluctuations, the floating potential can fall to zero. We revealed this in [4] and explained this effect in terms of increasing electron temperature. Owing to the fact that the directed velocity of ions in the cathode spot plasma considerably (by an order of magnitude) surpasses the thermal velocity of ions, we also discussed its influence on the probe indication, depending on its position in the plasma with respect to the ion jet. In the present work we verified this experimentally. Figure 6 shows waveforms from the floating probe for the two positions with respect to the cathode. When the probe is in the cathode shade (location 1), the floating potential falls periodically to zero or even becomes negative. At these moments electron current from the plasma can flow to the earthed cathode but its amplitude in the bursts is not in excess of $10-20 \mathrm{~mA}$ at arc current $\simeq 5-10 \mathrm{~A}$ [4]. When ion jets affect the probe (location 2), the latter indicates a higher floating potential. This is explained by a considerable excess of the directed ion current over the chaotic one. The error that this introduces leads to the measurements of plasma electron temperature being too low. Kutzner and Glinkovski have also discussed this effect [26].

At moments when the floating potential $V_{\mathrm{f}}=0$, hot electrons also should be taken into account while heating the cathode surface. The density of flux power in this case is [20]

$$
P=\frac{n_{\mathrm{i}}(k T)^{1 / 2}}{\left(2 \pi m_{\mathrm{i}}\right)^{3 / 2}}\left(\frac{1}{2} \ln \left(\frac{m_{\mathrm{i}}}{m_{\mathrm{e}}}\right)+4\right) .
$$

For $n_{\mathrm{j}}=10^{26} \mathrm{~m}^{-3}, T_{\mathrm{e}}=5 \mathrm{eV}, P=5 \times 10^{11} \mathrm{~W} \mathrm{~m}^{-2}$, that is, it is also rather high and can be higher at a distance of $\simeq 1 \mu \mathrm{m}$.

In [16], while estimating the electron current returning to the cathode, the potential $V_{\mathrm{p}}=V_{\mathrm{f}} \simeq 5 \mathrm{~V}$ was taken as the potential difference between the cathode and the plasma at a distance of $r=1 \mu \mathrm{m}$. This is an error. In fact, the plasma potential $V_{\mathrm{p}}$ is $V_{\mathrm{p}}=V_{\mathrm{f}}+\phi$. When $V_{\mathrm{f}}=0$, for $\mathrm{Cu}$ plasma $\phi=5.1 k T_{\mathrm{e}} / e$. (Plasma 


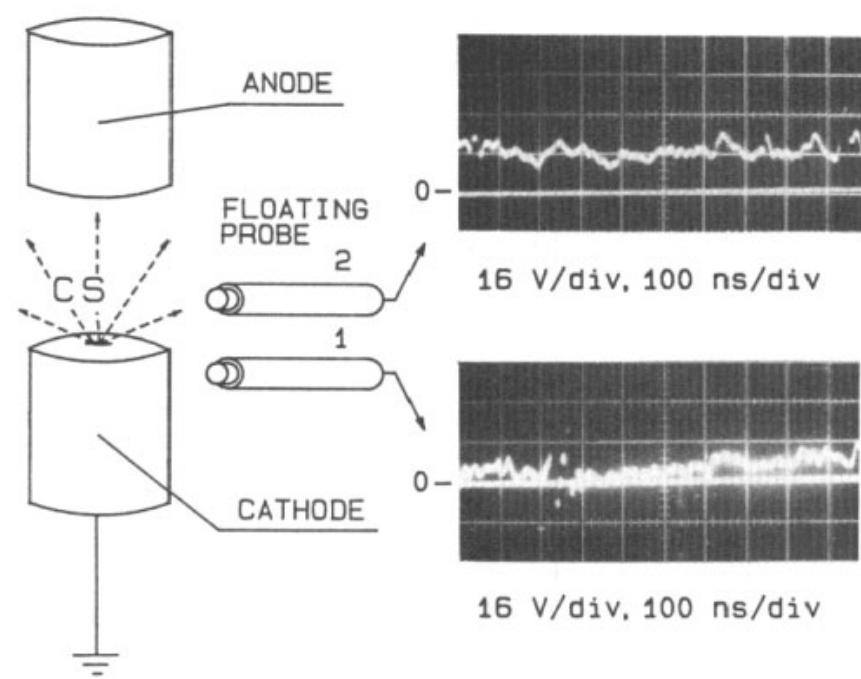

Figure 6. Oscillograms of the floating potential, illustrating the influence of probe location on its indication.

temperature at a distance of $r \simeq 1 \mu \mathrm{m}$ was assumed $\simeq 5 \mathrm{eV}$ at work [16]). Hence, at $k T_{\mathrm{e}}=4-5 \mathrm{eV}$ $\phi=20-25 \mathrm{~V}$. Then the reverse electron current density to the cathode, which is proportional to $\exp \left(-e \phi / k T_{\mathrm{e}}\right)$ will be much lower than that calculated in [8]. If the plasma potential increases continuously with radius, then $T_{\mathrm{e}}$ within $r=1-2 \mu \mathrm{m}$ cannot be higher than $1-2 \mathrm{eV}$, due to plasma cooling. Actually, a characteristic scale of heat diffusion into plasma [21] is given by

$$
\frac{r^{2}}{t}=4 \alpha^{2}=\frac{4 \kappa}{C_{V} \rho}
$$

where $\kappa, C_{V}$ and $\rho$ are respectively the heat conductivity, the heat capacity and the specific mass of the plasma. For $n_{\mathrm{i}}=10^{26} \mathrm{~m}^{-3}, k T_{\mathrm{e}}=5 \mathrm{eV}$, the plasma cools within $r=1 \mu \mathrm{m}$ in time $t=1 \mathrm{~ns}$, that is within the size of the spot. According to recent measurement [27], the plasma density within $r \leqslant 5-10 \mu \mathrm{m}$ from the spot is $n=10^{26} \mathrm{~m}^{-3}$. A low plasma temperature $(1-2 \mathrm{eV})$ near the cathode also follows from recent calculations [28].

Thus, experimental findings show strong evidence for the mechanism of new spot initiation by intensive surface heating (mainly by the ion flux). Near the footpoint of a spot the flux density may be still higher. Quite naturally, the inceptive craters become invisible, having been buried beneath the large crater left by the cathode spot. In the transverse magnetic field the spots draw up in a line parallel to field $H$ and inceptive craters at the wing sometimes become distinct (figure 1).

\subsection{Some remarks on the retrograde motion of the cathode spot}

One can find detailed information on the arc motion in the transverse $H$ field, for example, in a brief review [29]. Here we only emphasize the most general points.

We proceed from the fact that the efficiency of generation of new spots in the $\boldsymbol{H}$ field on the 'retrograde side' of the spot is higher than that on the 'direct side'.
Here a high efficiency is caused by a higher plasma density and therefore by a higher density of flux power on the cathode. This follows from calculation and experimental evidence $[29,30]$, and is determined by the anisotropy of plasma expansion velocity in the $H$ field.

The arc behaviour in the transverse magnetic field (as well as without a magnetic field) becomes more complicated due to the influence of surface contamination on arc characteristics. On a clean cathode surface the arc can be expected to move always in the retrograde direction at any ambient gas pressure, arc current and magnetic field, if the arc gap is small enough. For example, the retrograde motion of a $1000 \mathrm{~A}$ arc is maintained up to pressure 900 Torr in argon [31] and this can be explained by the clean surface conditioned by the arc itself. A similar behaviour persists at $100 \mathrm{~A}$ on a clean cathode at pressures up to 1500 Torr [32].

Non-metallic films and contaminations promote appearance of new spots at large distances (tens and hundreds of micrometres). Therefore, the efficiency of new spot initiation is higher under the plasma column (in the amperian direction), especially at higher pressures. Therefore, a small insulation obstruction on the cathode stops the arc when it moves in the retrograde direction, but only delays it in the amperian direction [33]. In fact, spot motion by small steps in the retrograde direction is interrupted by large jumps in the amperian one, therefore, resultant motion occurs in the amperian direction [32]. The initiation of new spots in the amperian direction can be intensified by the appearance of moving layers charged to high potential in the plasma column [19]. These layers are caused by mass flux fluctuations.

Some authors interpret the retrograde motion as attracting the Hall field [34]. It should be noted, however, that within distance $<10 \mu \mathrm{m}$, where new spots appear, the Hall parameter $\omega \tau<1$ [19], hence Hall addition to the value $U_{\mathrm{c}}$ is negligible.

\section{Conclusion}

New experiment evidence, combined with theoretical considerations, suggests strongly that a new cathode spot appears due to intensive heating of the cathode surface by the flux of charged particles from the plasma (mainly by ions) as a result of the increase in local plasma density. A high-energy plasma jet is generated within distance $<10 \mu \mathrm{m}$ from the cathode surface. The duration of these local microjets is $10-50$ ns.The flux power density exceeds $10^{12} \mathrm{~W} \mathrm{~m}^{-2}$ and is sufficient to heat the cathode surface during time $\sim 1 \mathrm{~ns}$.

Inversion of spot motion in transverse magnetic field from retrograde to direct seems to be caused by the influence of cathode surface contaminations on the efficiency of new spot initiation.

\section{References}

[1] Kesaev I G Cathode Processes of Electrical Arc (Moscow: Nauka) (English translation sand 78-6011 NTIS) 
[2] Lyubimov G A and Rakhovskii V I 1978 Usp. Fiz. Nauk 125 665-706 (English translation 1978 Sov. Phys. Usp. 21 693-718)

[3] Golub V N 1974 Experimental investigation rapidly moving arc cathode spots in tangential magnetic field with high spatial and temporal resolution Thesis High Temperature Institute, Moscow (in Russian)

[4] Puchkarev V F 1991 J. Phys. D: Appl. Phys. 24 685-92

[5] Proskurovskii D I and Puchkarev V F $1979 \mathrm{Zh}$. Tekh. Fiz. 49 2611-18 (English translational 1979 Sov. Phys. Tech. Phys. 24 1474-8)

[6] Lutz M A 1974 IEEE Trans. Plasma Sci. 2 1-24

[7] Jakubka K and Juttner B 1981 J. Nucl. Mater. 102 259-66

[8] McClure G W 1974 J. Appl. Phys. 45 2078-84

[9] Hantzsche E, Juttner B, Puchkarev V F et al $1976 \mathrm{~J}$. Phys. D: Appl. Phys. 9 1771-81

[10] Mesyats G A and Proskurovskii D I 1989 Pulse Electrical Discharge in Vacuum (Heidelberg: Springer)

[11] Juttner B 1979 Beitr. Plasma Phys. 19 25-48

[12] Puchkarev V F and Murzakayev A M 1990 J. Phys. D: Appl. Phys. 23 26-36

[13] Mesyats G A 1984 Proc. 11th Int. Symp. on Discharges and Electrical Insulation in Vacuum (Berlin: GDR) pp 93-100

[14] Ecker G 1980 Vacuum Arcs, Theory and Application ed J M Lafferty (New York: Wiley)

[15] Nemchinskii V A 1983 Zh. Tekh. Fiz. 53 235-40 (English translation Sov. Phys. - Tech. Phys. 28 150-5)

[16] Litvinov E A, Mesyats G A and Parfyonov A G 1990 Dokl Acad. Nauk USSR 310 344-48 (English translation 1990 Sov. Phys. Dokl. 35 47-8)

[17] Juttner B, Pursch $\mathbf{H}$ and Anders S 1984 J. Phys. D: Appl. Phys. 17 L111-4

[18] Kutzner J and Miller H C 1989 IEEE Trans. Plasma Sci.
17 688-94

[19] Proskurovskii D I and Puchkarev V F $1980 \mathrm{Zh}$. Tekh. Fiz. 50 2120-6 (English translation Sov. Phys. - Tech. Phys. 25 1235-9)

[20] Puchkarev V F and Chesnokov S M 1992 J. Phys. D: Appl. Phys. 25 1760-6

[21] Lovberg R H 1968 Plasma Diagnostic Technique ed R H Haudllstone and Leonard (New York: Academic)

[22] Lyubimov G A 1975 Dokl. Acad. Nauk USSR 225 1045-8 (English translation Sov. Phys. - Dokl. 20 830-2)

[23] Wieckert C 1987 Contrib. Plasma Phys. 27 309-30

[24] Hantzsche E 1991 J. Phys. D: Appl. Phys. 24

[25] Korop E D 1976 Zh. Tekh. Fiz. 46 2187-90 (English translation Sov. Phys. - Tech. Phys. 21 1284-6)

[26] Kutzner J and Glinkowski M 1983 IEEE Trans. Plasma Sci. 11 233-8

[27] Anders A, Anders S, Juttner B et al 1992 IEEE Trans. Plasma Sci. 20 466-72

[28] Nemirovskii A Z, Puchkarev V F and Tarbeev O I Proc. 15th Int. Symp. Discharges and Electrical Insulators in Vacuum (Darmstadt) pp 339-44

[29] Drouet M G 1984 Proc. 11th Int. Symp. on Discharges and Electrical Insulators in Vacuum (East Berlin) pp 107-14

[30] Emtage P R, Corman J G, Heberlein J V R, Holmes F A et al 1977 Proc. 13th Int. Conf. on Phenomena in lonized Gases (East Berlin) p 676

[31] Carter R P and Murphree D L 1973 J. Appl. Phys. 44 5190-1

[32] Juttner B 1985 J. Phys. D: Appl. Phys. 18 2221-31

[33] Roman W C 1963 Proc. VI lnt. Conf. Phenomena in Ionized Gases p 287

[34] Moizhes B Ya and Nemchinskii V A 1991 J. Phys. D: Appl. Phys. 24 2014-19 